\title{
Erratum: Simple self-interaction correction to random-phase-approximation-like correlation energies [Phys. Rev. A 100, 022515 (2019)]
}

\author{
Tim Gould, Adrienn Ruzsinszky, and John P. Perdew
}

(Received 5 January 2020; published 13 May 2020)

DOI: 10.1103/PhysRevA.101.059902

Our recent work introduced a theoretical metageneralized gradient approximation based correction (gRPA+) to randomphase approximation (RPA) calculations that improved absolute correlation energies, and energy differences, compared to RPA and locally corrected RPA (RPA+). Unfortunately, the RPA+ and gRPA+ data for open-shell systems reported in our work were incorrect; the former substantially and the latter only slightly. This error was caused by incorrect implementation of the spin-polarized local random-phase approximation (LRPA), which led to errors in RPA + and through it gRPA+.

Here, we reanalyze the data using a correct implementation of LRPA.

Firstly, we note that our RPA calculations were done with reference Kohn-Sham eigenvalues and eigenfunctions from a spherically symmetric exact-exchange potential. The more standard RPA with a Perdew-Burke-Ernzerhof reference could slightly modify the results.

Our work contained two figures and three tables, and reported results for two versions of gRPA,+ denoted gRPA $+\left(g_{1}\right)$ and gRPA $+\left(g_{2}\right)$. All results contain errors. Here, we present updated versions using correct data. Tables and figures are numbered per the original paper, for ease of comparison.

gRPA + involved an optimization process to determine an optimized parameter $c_{q}^{\text {opt }}$, where $c_{1}^{\text {opt }}$ was restricted to be an integer, while $c_{2}^{\text {opt }}$ was restricted to be "reasonable." After correction, both values were found to be the same as previously reported, due to these constraints. Similarities to previous work can be seen by comparing the updated Fig. 1 reported here with the left panel of Fig. 1 of the published article.

The corrected RPA+ correlation energies for ionization potentials $\mathrm{IP}_{c}$ (Table I) now have a mean absolute error (MAE) that is significantly reduced to $4.3 \mathrm{kcal} / \mathrm{mol}$, from $8.6 \mathrm{kcal} / \mathrm{mol}$ previously. gRPA + then offers improvements of around $30 \%$, yielding MAEs of $3.6 \mathrm{kcal} / \mathrm{mol}\left(g_{1}\right.$; was 3.5$)$ and $3.8 \mathrm{kcal} / \mathrm{mol}\left(g_{2}\right.$; was 3.6$)$.

The situation for anions (Table II) is similar. There, we find MAEs: $5.4 \mathrm{kcal} / \mathrm{mol}$ (RPA+; was 8.1$), 4.1 \mathrm{kcal} / \mathrm{mol}\left(g_{1}\right.$; was 3.9), and $4.3 \mathrm{kcal} / \mathrm{mol}\left(g_{2}\right.$; was 4.2$)$.

Overall, gRPA+ improves correlations for most species (see Fig. 2 and Table III) and improves the MAE from 108.8 kcal/mol (RPA) or $7.4 \mathrm{kcal} / \mathrm{mol}(\mathrm{RPA}+$; was 7.9$)$ to $6.3 \mathrm{kcal} / \mathrm{mol}\left(g_{1}\right.$ or $g_{2}$; was 6.2$)$. We note that additional scrutiny of the original article also revealed a more minor error, in that Table III showed correlation energies only from one of the two gRPA + forms. This has also been corrected here.

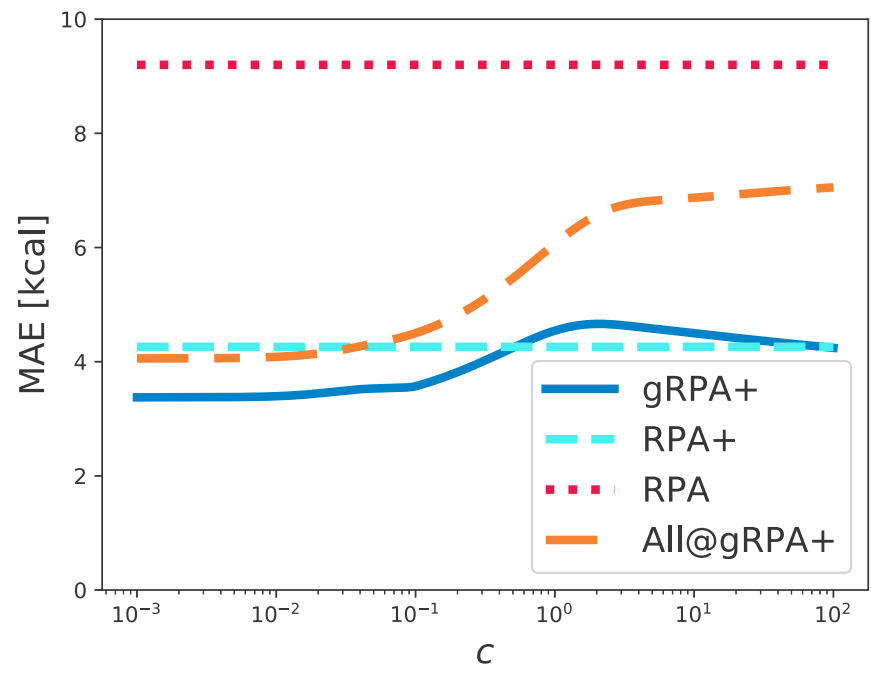

FIG. 1. MAE of IPs as a function of $c_{2}$, for RPA, RPA+, and gRPA+ (also shown, MAE for all correlation energies). 
TABLE I. Correlation contributions $\mathrm{IP}_{c}$ to ionization potentials IP and their errors for RPA, RPA + , and gRPA + using $c_{1}^{\mathrm{opt}}=1$ and $c_{2}^{\mathrm{opt}}=$ 0.2 . All energies in $\mathrm{kcal} / \mathrm{mol}$. Mean absolute errors (MAEs) are also reported.

\begin{tabular}{|c|c|c|c|c|c|}
\hline \multirow[b]{2}{*}{ Element } & \multirow{2}{*}{$\begin{array}{c}\mathrm{IP}_{c} \\
\text { Exact }\end{array}$} & \multicolumn{4}{|c|}{ Error } \\
\hline & & RPA & $\mathrm{RPA}+$ & $g \mathrm{RPA}+\left(g_{1}\right)$ & $g \mathrm{RPA}+\left(g_{2}\right)$ \\
\hline $\mathrm{Be}$ & 29.4 & 8.2 & -1.0 & 5.8 & 5.8 \\
\hline $\mathrm{C}$ & 11.0 & 8.1 & -3.2 & -5.2 & -5.1 \\
\hline $\mathrm{N}$ & 13.6 & 15.3 & 3.6 & 1.7 & 2.4 \\
\hline $\mathrm{O}$ & 40.2 & -0.9 & -11.7 & -6.6 & -6.9 \\
\hline $\mathrm{F}$ & 40.2 & 9.1 & -2.2 & -0.1 & -0.5 \\
\hline $\mathrm{Na}$ & 3.8 & 13.5 & 3.7 & 2.1 & 2.3 \\
\hline MAE & & 9.2 & 4.3 & 3.6 & 3.8 \\
\hline
\end{tabular}

TABLE II. Correlation contributions $\mathrm{EA}_{c}$ to electron affinities EA and their errors for RPA, RPA + , and gRPA + using $c_{1}^{\mathrm{opt}}=1$ and $c_{2}^{\mathrm{opt}}=$ 0.2. All energies in $\mathrm{kcal} / \mathrm{mol}$. Mean absolute errors (MAEs) are also reported.

\begin{tabular}{|c|c|c|c|c|c|}
\hline \multirow[b]{2}{*}{ Element } & \multirow{2}{*}{$\begin{array}{r}\text { EA }_{c} \\
\text { Exact }\end{array}$} & \multicolumn{4}{|c|}{ Error } \\
\hline & & RPA & $\mathrm{RPA}+$ & $g \mathrm{RPA}+\left(g_{1}\right)$ & $g \mathrm{RPA}+\left(g_{2}\right)$ \\
\hline $\mathrm{C}$ & 16.6 & 16.1 & 5.5 & 3.7 & 4.2 \\
\hline $\mathrm{N}$ & 44.7 & -1.3 & -11.1 & -6.6 & -6.9 \\
\hline $\mathrm{O}$ & 45.9 & 8.1 & -2.4 & -0.3 & -0.7 \\
\hline $\mathrm{F}$ & 47.1 & 17.0 & 5.9 & 6.5 & 6.4 \\
\hline $\mathrm{Na}$ & 15.0 & 9.7 & 1.9 & 3.5 & 3.4 \\
\hline MAE & & 10.4 & 5.4 & 4.1 & 4.3 \\
\hline
\end{tabular}
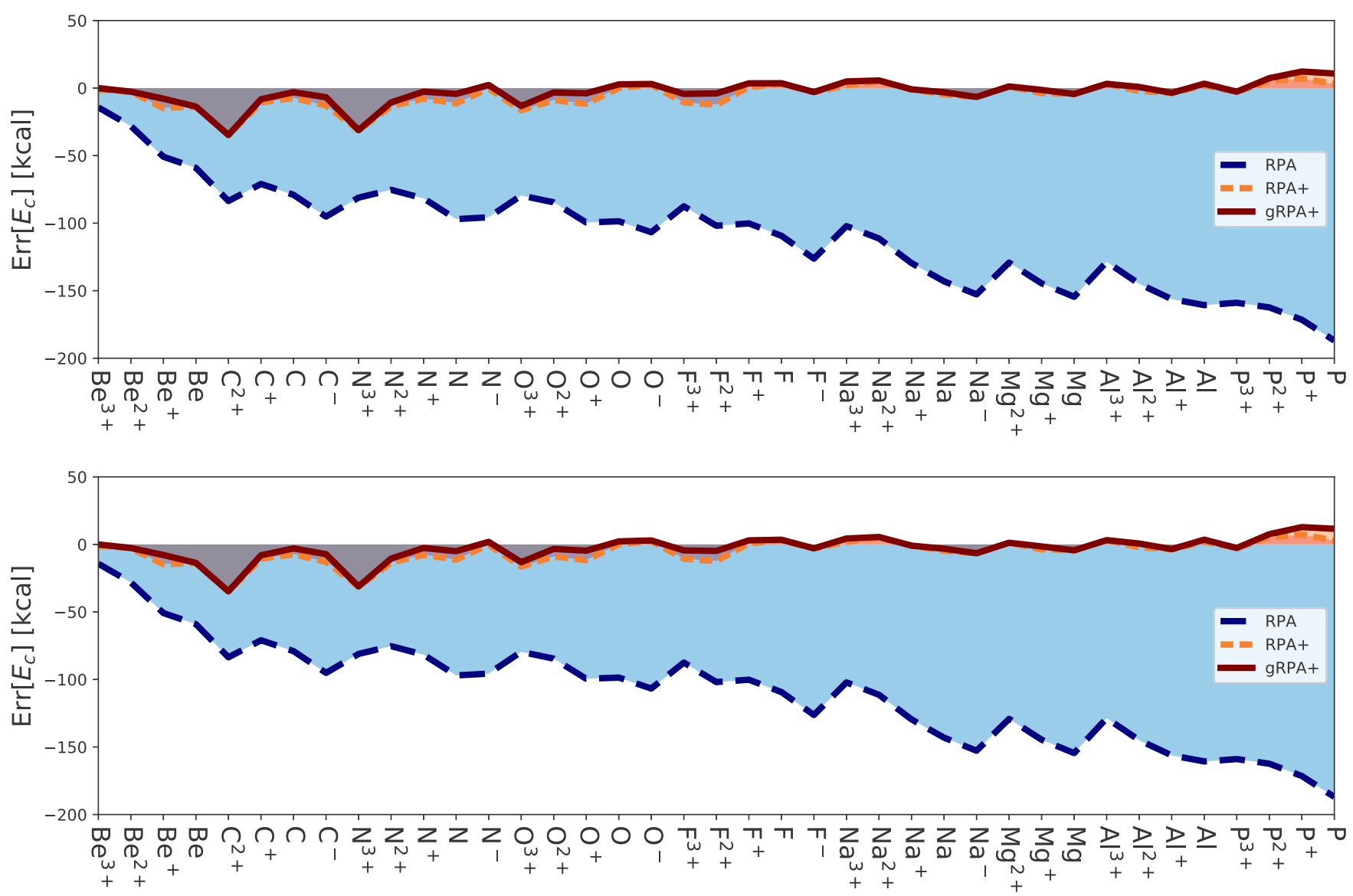

FIG. 2. Correlation energy errors $(\mathrm{kcal} / \mathrm{mol})$ for the full set of atoms and ions, using the models $g_{1}$ (top) and $g_{2}$ (bottom) [i.e., using Eqs. (8a) and (8b) in Eq. (6) of our original paper, with $c_{1}^{\text {opt }}=1$ and $c_{2}^{\text {opt }}=0.2$ ]. Shown are gRPA+ (solid red curve), RPA+ (short-dashed orange curve and shading), and RPA (long-dashed blue curve and shading). 
TABLE III. Correlation energy $E_{c}$ and errors for RPA, RPA + , and gRPA + under both models, with $c_{1}^{\text {opt }}=1$ and $c_{2}^{\text {opt }}=0.2$. Unlike our original work, we include total correlation energies using both flavors of gRPA + . All energies in $\mathrm{kcal} / \mathrm{mol}$.

\begin{tabular}{|c|c|c|c|c|c|c|c|c|c|}
\hline & \multirow[b]{2}{*}{$Z$} & \multirow[b]{2}{*}{$N$} & \multicolumn{3}{|c|}{$E_{c}$} & \multicolumn{4}{|c|}{ Error } \\
\hline & & & Exact & $g \mathrm{RPA}+\left(g_{1}\right)$ & $g \mathrm{RPA}+\left(g_{2}\right)$ & RPA & RPA+ & $g \mathrm{RPA}+\left(g_{1}\right)$ & $g \mathrm{RPA}+\left(g_{2}\right)$ \\
\hline $\mathrm{Be}^{+3}$ & 4 & 1 & 0.0 & 0.0 & 0.0 & -14.3 & -1.1 & 0.0 & 0.0 \\
\hline $\mathrm{Be}^{+2}$ & 4 & 2 & -27.6 & -30.3 & -30.3 & -28.1 & -2.6 & -2.6 & -2.6 \\
\hline $\mathrm{Be}^{+}$ & 4 & 3 & -29.7 & -37.6 & -37.6 & -50.8 & -14.7 & -7.9 & -7.9 \\
\hline $\mathrm{Be}$ & 4 & 4 & -59.2 & -72.9 & -72.9 & -59.1 & -13.7 & -13.7 & -13.7 \\
\hline $\mathrm{C}^{+2}$ & 6 & 4 & -69.9 & -104.6 & -104.6 & -83.6 & -34.8 & -34.8 & -34.8 \\
\hline $\mathrm{C}^{+}$ & 6 & 5 & -87.1 & -95.3 & -95.2 & -71.0 & -10.6 & -8.2 & -8.1 \\
\hline $\mathrm{C}$ & 6 & 6 & -98.2 & -101.2 & -101.2 & -79.0 & -7.3 & -3.0 & -3.0 \\
\hline $\mathrm{C}^{-}$ & 6 & 7 & -114.8 & -121.5 & -122.0 & -95.1 & -12.8 & -6.8 & -7.2 \\
\hline $\mathrm{N}^{+3}$ & 7 & 4 & -88.2 & -119.3 & -119.3 & -81.1 & -31.2 & -31.2 & -31.2 \\
\hline $\mathrm{N}^{+2}$ & 7 & 5 & -94.5 & -105.1 & -105.0 & -75.3 & -13.4 & -10.6 & -10.5 \\
\hline $\mathrm{N}^{+}$ & 7 & 6 & -104.6 & -107.2 & -107.2 & -81.7 & -7.7 & -2.6 & -2.6 \\
\hline $\mathrm{N}$ & 7 & 7 & -118.2 & -122.5 & -123.2 & -97.0 & -11.3 & -4.3 & -5.0 \\
\hline $\mathrm{N}^{-}$ & 7 & 8 & -162.9 & -160.6 & -161.0 & -95.7 & -0.2 & 2.3 & 1.9 \\
\hline $\mathrm{O}^{+3}$ & 8 & 5 & -101.0 & -114.3 & -114.2 & -79.6 & -16.4 & -13.3 & -13.2 \\
\hline $\mathrm{O}^{+2}$ & 8 & 6 & -109.8 & -113.1 & -113.2 & -84.6 & -9.0 & -3.2 & -3.3 \\
\hline $\mathrm{O}^{+}$ & 8 & 7 & -121.8 & -125.6 & -126.4 & -99.5 & -11.6 & -3.9 & -4.7 \\
\hline $\mathrm{O}$ & 8 & 8 & -161.9 & -159.2 & -159.7 & -98.6 & 0.1 & 2.7 & 2.2 \\
\hline $\mathrm{O}^{-}$ & 8 & 9 & -207.8 & -204.7 & -204.9 & -106.7 & 2.5 & 3.1 & 2.9 \\
\hline $\mathrm{F}^{+3}$ & 9 & 6 & -114.2 & -118.5 & -118.7 & -87.5 & -10.6 & -4.3 & -4.4 \\
\hline $\mathrm{F}^{+2}$ & 9 & 7 & -124.9 & -128.9 & -129.7 & -101.9 & -12.4 & -4.0 & -4.9 \\
\hline $\mathrm{F}^{+}$ & 9 & 8 & -163.8 & -160.3 & -160.8 & -100.2 & 0.8 & 3.5 & 3.0 \\
\hline $\mathrm{F}$ & 9 & 9 & -204.0 & -200.4 & -200.5 & -109.3 & 3.0 & 3.6 & 3.5 \\
\hline $\mathrm{F}^{-}$ & 9 & 10 & -251.0 & -254.0 & -254.0 & -126.3 & -2.9 & -2.9 & -2.9 \\
\hline $\mathrm{Na}^{+3}$ & 11 & 8 & -168.2 & -163.3 & -163.8 & -102.2 & 1.9 & 4.9 & 4.4 \\
\hline $\mathrm{Na}^{+2}$ & 11 & 9 & -205.2 & -199.6 & -199.7 & -111.3 & 5.0 & 5.7 & 5.5 \\
\hline $\mathrm{Na}^{+}$ & 11 & 10 & -244.1 & -245.1 & -245.1 & -129.6 & -0.9 & -0.9 & -0.9 \\
\hline $\mathrm{Na}$ & 11 & 11 & -247.9 & -251.0 & -251.1 & -143.1 & -4.7 & -3.1 & -3.2 \\
\hline $\mathrm{Na}^{-}$ & 11 & 12 & -262.9 & -269.5 & -269.5 & -152.8 & -6.6 & -6.6 & -6.6 \\
\hline $\mathrm{Mg}^{+2}$ & 12 & 10 & -244.8 & -243.5 & -243.5 & -129.2 & 1.2 & 1.2 & 1.2 \\
\hline $\mathrm{Mg}^{+}$ & 12 & 11 & -251.0 & -252.4 & -252.5 & -144.6 & -3.7 & -1.3 & -1.5 \\
\hline $\mathrm{Mg}$ & 12 & 12 & -274.9 & -279.3 & -279.3 & -154.5 & -4.4 & -4.4 & -4.4 \\
\hline $\mathrm{Al}^{+3}$ & 13 & 10 & -245.4 & -242.2 & -242.2 & -128.7 & 3.2 & 3.2 & 3.2 \\
\hline $\mathrm{Al}^{+2}$ & 13 & 11 & -254.2 & -253.4 & -253.5 & -145.0 & -2.1 & 0.8 & 0.6 \\
\hline $\mathrm{Al}^{+}$ & 13 & 12 & -283.7 & -287.2 & -287.2 & -156.2 & -3.5 & -3.5 & -3.5 \\
\hline $\mathrm{Al}$ & 13 & 13 & -295.0 & -291.7 & -291.5 & -160.7 & 2.0 & 3.3 & 3.5 \\
\hline $\mathrm{P}^{+3}$ & 15 & 12 & -296.2 & -298.9 & -298.9 & -159.0 & -2.7 & -2.7 & -2.7 \\
\hline $\mathrm{P}^{+2}$ & 15 & 13 & -313.2 & -305.8 & -305.6 & -162.4 & 5.1 & 7.4 & 7.6 \\
\hline $\mathrm{P}^{+}$ & 15 & 14 & -327.6 & -315.3 & -314.8 & -171.5 & 7.3 & 12.3 & 12.8 \\
\hline \multirow[t]{2}{*}{$\mathrm{P}$} & 15 & 15 & -338.9 & -328.1 & -327.3 & -187.0 & 3.0 & 10.8 & 11.6 \\
\hline & MAE & & & & & 108.8 & 7.4 & 6.3 & 6.3 \\
\hline
\end{tabular}

As a result of the reanalysis, we find that the gains from RPA + to gRPA+ are more muted compared to our initially published work. gRPA+ offers more limited advantages over RPA+, of $20 \%-30 \%$ or a bit over $1 \mathrm{kcal} / \mathrm{mol}$ for the correlation contribution to ionization potentials and electron affinities. This could presumably be improved by further refinement of the model.

However, as a result of our error, we expose an additional advantage of gRPA+ over RPA and RPA+. gRPA+ correctly zeros out the correlation energy density in one-electron regions, and scales it down in some other spin-polarized regions. These regions can cause problems for convergence when using localized atomic basis sets, due to "basis set incompleteness errors" [1] (BSIE), 
and can also suffer related problems arising from poor ground-state orbitals [2]. Thus, gRPA+ may be easier to converge within a localized basis set than RPA by "screening out" the BSIE and errors caused by poor orbitals. (Note, in our work we use a grid so are unable to test this assertion). This will be explored in future work.

The authors acknowledge helpful discussions with Shiqi Ruan and Filipp Furche.

[1] D. Sherrill, Distinguishing basis set superposition error (BSSE) from basis set incompleteness error (BSIE), http://vergil.chemistry.gatech.edu/notes/bsse-vs-bsie.pdf (accessed 04/04/2020).
[2] V. K. Voora, S. G. Balasubramani, and F. Furche, Phys. Rev. A 99, 012518 (2019). 